\title{
Well-posedness for the heat flow of polyharmonic maps with rough initial data
}

\author{
Tao Huang* Changyou Wang*
}

\begin{abstract}
We establish both local and global well-posedness of the heat flow of polyharmonic maps from $\mathbb{R}^{n}$ to a compact Riemannian manifold without boundary for initial data with small BMO norms.
\end{abstract}

\section{Introduction}

For $k \geq 1$, let $N$ be a $k$-dimensional compact Riemannian manifold without boundary, isometrically embedded in some Euclidean space $\mathbb{R}^{l}$. For $n \geq 2$ and $m \geq 1$, we consider the $m$-th order energy functional

$$
E_{m}(u)=\frac{1}{2} \int_{\mathbb{R}^{n}}\left|\nabla^{m} u\right|^{2}= \begin{cases}\frac{1}{2} \int_{\mathbb{R}^{n}}\left|\Delta^{\frac{m}{2}} u\right|^{2} & \text { if } m \text { is even } \\ \frac{1}{2} \int_{\mathbb{R}^{n}}\left|\nabla \Delta^{\frac{m-1}{2}} u\right|^{2} & \text { if } m \text { is odd }\end{cases}
$$

for any $u \in W^{m, 2}\left(\mathbb{R}^{n}, N\right)$, where $\Delta$ is the Laplace operator on $\mathbb{R}^{n}$ and

$$
W^{m, 2}\left(\mathbb{R}^{n}, N\right)=\left\{v \in W^{m, 2}\left(\mathbb{R}^{n}, \mathbb{R}^{l}\right): v(x) \in N \text { for a.e. } x \in \Omega\right\} .
$$

Recall that a map $u \in W^{m, 2}\left(\mathbb{R}^{n}, N\right)$ is called a polyharmonic map if $u$ is the critical point of $E_{m}$. The Euler-Lagrange equation of polyharmonic maps is (see Gastel-Scheven [9]):

$$
\begin{aligned}
(-1)^{m} \Delta^{m} u=F(u):= & (-1)^{m} \operatorname{div}^{m}\left(\sum_{k=0}^{m-2}\left(\begin{array}{c}
m-1 \\
k
\end{array}\right) \nabla^{m-k-1}(\Pi(u)) \nabla^{k+1} u\right) \\
& -\sum_{k=0}^{m-1}(-1)^{k}\left(\begin{array}{c}
m \\
k
\end{array}\right) \operatorname{div}^{k}\left(\nabla^{m-k}(\Pi(u)) \nabla^{m} u\right)
\end{aligned}
$$

where $\Pi: N_{\delta} \rightarrow N$ is the nearest point projection from the $\delta$-neighborhood of $N$ to $N$, which is smooth provide $\delta=\delta(N)>0$ is sufficiently small. It is readily seen that (1.1) becomes the equation of harmonic maps for $m=1$, and of extrinsic biharmonic maps for $m=2$.

Motivated by the study of heat flow of harmonic and biharmonic maps, we consider the heat flow of polyharmonic maps, i.e. $u: \mathbb{R}^{n} \times \mathbb{R}_{+} \rightarrow N$ solves

${ }^{*}$ Department of Mathematics, University of Kentucky, Lexington, KY 40506 


$$
\begin{aligned}
u_{t}+(-1)^{m} \Delta^{m} u & =F(u) & & \text { in } \mathbb{R}^{n} \times(0,+\infty) \\
\left.u\right|_{t=0} & =u_{0} & & \text { on } \mathbb{R}^{n}
\end{aligned}
$$

where $u_{0}: \mathbb{R}^{n} \rightarrow N$ is a given map.

The heat flow of harmonic maps, (1.2) for $m=1$, has been extensively studied. For smooth initial data, the existence of global smooth heat flow of harmonic maps has been established by (i) Eells-Sampson [7] under the assumption that the sectional curvature $K_{N} \leq 0$, and (ii) Hildebrandt-Kaul-Widman [11] under the assumption that the image of $u_{0}$ is contained in a geodesic ball $B_{R}$ in $N$ with radius $R<\frac{\pi}{\sqrt[2]{\sqrt{\max _{R}\left|K_{N}\right|}}}$. In general, the short time smooth heat flow of harmonic maps may develop singularity at finite time, see Coron-Ghidaglia [4], Chen-Ding [2], and Chang-Ding-Ye [3]. However, Chen-Struwe [6] (see also Chen-Lin [5] and Lin-Wang [19]) proved the existence of partially smooth, global weak solutions to (1.2)-(1.3) for smooth initial data $u_{0}$. For rough initial data $u_{0}$, the second author recently proved in 25] the well-posedness for the heat flow of harmonic maps provided the BMO norm of $u_{0}$ is small.

When $m=2$, (1.2) becomes the heat flow of extrinsic biharmonic maps, which was first studied by Lamm in [15, 16, 17. In particular, it was proven in [15, 16, 17] that if $n=4$ and $\left\|u_{0}\right\|_{W^{2,2}\left(\mathbb{R}^{4}\right)}$ is sufficiently small, then there exists a unique global smooth solution. For an arbitrary $u_{0} \in W^{m, 2}\left(\mathbb{R}^{2 m}\right)$, it was later independently proved by Wang [23] (for $m=2$ ) and Gastel [8] (for $m \geq 2$ ) that there exists a global weak solution to (1.2)-(1.3) that is smooth away from finitely many singular times. Very recently, the second author established in 24] the well-posedness for the heat flow of biharmonic maps for $u_{0}$ with small BMO norm.

We would like to mention that there have been some works on the regularity of polyharmonic maps for $m \geq 3$ in the critical dimensions $n=2 m$. We refer the readers to Gastel-Scheven [9], Lamm-Wang [18, Goldstein-Strzelecki-Zatorska-Goldstein[10], Moser [20], and Angelsberg-Pumberger [1].

In this paper, we are interested in the well-posedness of the heat flow of polyharmonic maps with rough initial data. In particular, we aim to extend the techniques from [25, 24] to establish the well-posedness of the heat flow of polyharmonic maps (1.2) and (1.3) for $m \geq 3$ with $u_{0}$ having small BMO norm.

We remark that the techniques employed by Wang [25, 24] were motivated by the earlier work by Koch and Tataru [14] on the global well-posedness of the incompressible Navier-Stokes equation, and the recent work by Koch-Lamm [13] on geometric flows with rough initial data.

We first recall the BMO spaces. For $x \in \mathbb{R}^{n}$ and $r>0$, let $B_{r}(x) \subset \mathbb{R}^{n}$ be the ball with center $x$ and radius $r$. For $f: \mathbb{R}^{n} \rightarrow \mathbb{R}$, let $f_{x, r}$ be the average of $f$ over $B_{r}(x)$.

Definition 1.1 For $f: \mathbb{R}^{n} \rightarrow \mathbb{R}$ and $R>0$, define

$$
\mathrm{BMO}_{R}\left(\mathbb{R}^{n}\right)=\left\{f: \mathbb{R}^{n} \rightarrow \mathbb{R}\left|[f]_{\mathrm{BMO}\left(\mathbb{R}^{n}\right)}:=\sup _{x \in \mathbb{R}^{n}, 0<r \leq R} r^{-n} \int_{B_{r}(x)}\right| f-f_{x, r} \mid<+\infty\right\} .
$$

When $R=+\infty$, we simply write $\mathrm{BMO}\left(\mathbb{R}^{n}\right)$ for $\mathrm{BMO}_{\infty}\left(\mathbb{R}^{n}\right)$. 
For $0<T \leq \infty$, define the functional space $X_{T}$ by

$$
X_{T}:=\left\{f: \mathbb{R}^{n} \times[0, T] \rightarrow \mathbb{R}^{l} \mid\|f\|_{X_{T}}:=\sup _{0<t \leq T}\|f\|_{L^{\infty}\left(\mathbb{R}^{n}\right)}+[f]_{X_{T}}\right\},
$$

where

$$
[f]_{X_{T}}=\sum_{k=1}^{m}\left\{\sup _{0<t \leq T} t^{\frac{k}{2 m}}\left\|\nabla^{k} f\right\|_{L^{\infty}\left(\mathbb{R}^{n}\right)}+\sup _{x \in \mathbb{R}^{n}, 0<r \leq T}{ }_{\frac{1}{2 m}}\left(r^{-n} \int_{P_{r}\left(x, r^{2 m}\right)}\left|\nabla^{k} f\right|^{\frac{2 m}{k}}\right)^{\frac{k}{2 m}}\right\}
$$

where $P_{r}\left(x, r^{2 m}\right)=B_{r}(x) \times\left[0, r^{2 m}\right]$. It is clear that $\left(X_{T},\|\cdot\|_{X_{T}}\right)$ is a Banach space. When $T=+\infty$, we simply write $X,\|\cdot\|_{X}$, and $[\cdot]_{X}$ for $X_{\infty},\|\cdot\|_{X_{\infty}}$, and $[\cdot]_{X_{\infty}}$ respectively.

The main theorem is

Theorem 1.2 There exists an $\varepsilon_{0}>0$ such that for any $R>0$ if $u_{0}: \mathbb{R}^{n} \rightarrow N$ has

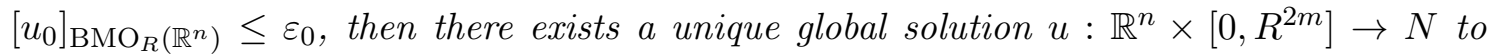
(1.2) and (1.3) with small semi-norm $[u]_{X_{R^{2 m}}}$.

As a direct consequence, we have

Corollary 1.3 There exists an $\varepsilon_{0}>0$ such that if $u_{0}: \mathbb{R}^{n} \rightarrow N$ has $\left[u_{0}\right]_{\mathrm{BMO}\left(\mathbb{R}^{n}\right)} \leq \varepsilon_{0}$, then there exists a unique global solution $u: \mathbb{R}^{n} \times \mathbb{R}_{+} \rightarrow N$ to (1.2) and (1.3) with small semi-norm $[u]_{X}$.

We follow the arguments in [25, 24] very closely. The paper is written as follows. In section 2, we present some basic estimates on the polyharmonic heat kernel. In section 3 , we present some crucial estimates on the polyharmonic heat equation. In section 4, we prove Theorem 1.2.

\section{The polyharmonic heat kernel}

In this section, we will prove some basic properties on the polyharmonic heat kernel.

The fundamental solution of the polyharmonic heat equation:

$$
b_{t}(x, t)+(-1)^{m} \Delta^{m} b(x, t)=0 \text { in } \mathbb{R}^{n} \times \mathbb{R}_{+}
$$

is given by

$$
b(x, t)=t^{-\frac{n}{2 m}} g\left(\frac{x}{t^{\frac{1}{2 m}}}\right)
$$

where

$$
g(x)=(2 \pi)^{-\frac{n}{2}} \int_{\mathbb{R}^{n}} e^{i x \cdot \xi-|\xi|^{2 m}} d \xi, x \in \mathbb{R}^{n} .
$$

It is easy to see that $g$ is smooth, radial, and

Proposition 2.1 For any $L \geq 0, k \geq 0$, there exists $C=C(k, L)>0$ such that

$$
\left|\nabla^{k} g(x)\right| \leq C(1+|x|)^{-L}, \forall x \in \mathbb{R}^{n} .
$$


Proof. For $k \geq 0$ and $L \geq 0$, since

$$
\nabla_{x}^{k}\left(e^{i x \cdot \xi}\right)=(i \xi)^{k}(i x)^{-L} \nabla_{\xi}^{L}\left(e^{i x \cdot \xi}\right)
$$

we have, by integration by parts,

$$
\begin{aligned}
\left|\nabla^{k} g(x)\right| & =\left|\int_{\mathbb{R}^{n}}(i x)^{-L} e^{i x \cdot \xi} \nabla_{\xi}^{L}\left((i \xi)^{k} e^{-|\xi|^{2 m}}\right) d \xi\right| \\
& \leq C(k, L)(1+|x|)^{-L} .
\end{aligned}
$$

This completes the proof.

As a direct consequence of (2.4), we have the following properties for the polyharmonic heat kernel $b$

Lemma 2.2 For any $k, L \geq 0$, there exist $C_{1}>0$ depending on $n, L$ and $C_{2}, C_{3}>0$ depending on $n, k, L$ such that for any $x \in \mathbb{R}^{n}$ and $t>0$, it holds:

$$
\begin{gathered}
|b(x, t)| \leq C_{1} t^{-\frac{n}{2 m}}\left(1+\frac{|x|}{t^{\frac{1}{2 m}}}\right)^{-L}, \\
\left|\nabla^{k} b(x, t)\right| \leq C_{2}\left(t^{-\frac{1}{2 m}}\right)^{n+k-L}\left(t^{\frac{1}{2 m}}+|x|\right)^{-L}, \\
\left\|\nabla^{k} b(x, t)\right\|_{L^{1}\left(\mathbb{R}^{n}\right)} \leq C_{3} t^{-\frac{k}{2 m}} .
\end{gathered}
$$

At the end of this section, we recall that the solution to the Dirichlet problem of inhomogeneous polyharmonic heat equation

$$
\begin{aligned}
u_{t}(x, t)+(-1)^{m} \Delta^{m} u(x, t) & =f(x, t) \text { in } \mathbb{R}^{n} \times \mathbb{R}_{+}, \\
u(x, 0) & =u_{0}(x) \text { on } \mathbb{R}^{n}
\end{aligned}
$$

is given by the following Duhamel formula:

$$
u=\mathbf{G} u_{0}+\mathbf{S} f
$$

where

$$
\mathbf{G} u_{0}(x, t):=\int_{\mathbb{R}^{n}} b(x-y, t) u_{0}(y) d y,(x, t) \in \mathbb{R}^{n} \times \mathbb{R}_{+},
$$

and

$$
\mathbf{S} f(x, t):=\int_{0}^{t} \int_{\mathbb{R}^{n}} b(x-y, t-s) f(y, s) d y d s,(x, t) \in \mathbb{R}^{n} \times \mathbb{R}_{+} .
$$




\section{Basic estimates for the polyharmonic heat equation}

In this section, we will provide some crucial estimates for the solution of the polyharmonic heat equation with initial data in BMO spaces.

Lemma 3.1 For $0<R \leq+\infty$, if $u_{0} \in \mathrm{BMO}_{R}\left(\mathbb{R}^{n}\right)$, then $\hat{u}_{0}:=\mathbf{G} u_{0}$ satisfies

$$
\sum_{k=1}^{m} \sup _{x \in \mathbb{R}^{n}, 0<r \leq R} r^{-n} \int_{P_{r}\left(x, r^{2 m}\right)} r^{2 k-2 m}\left|\nabla^{k} \hat{u}_{0}\right|^{2} \leq C\left[u_{0}\right]_{\mathrm{BMO}_{R}\left(\mathbb{R}^{n}\right)}^{2},
$$

and

$$
\sum_{k=1}^{m} \sup _{0<t \leq R^{2 m}} t^{\frac{k}{2 m}}\left\|\nabla^{k} \hat{u}_{0}(t)\right\|_{L^{\infty}\left(\mathbb{R}^{n}\right)} \leq C\left[u_{0}\right]_{\mathrm{BMO}_{R}\left(\mathbb{R}^{n}\right)}
$$

If, in addition, $u_{0} \in L^{\infty}\left(\mathbb{R}^{n}\right)$ then

$$
\sum_{k=1}^{m-1} \sup _{x \in \mathbb{R}^{n}, 0<r \leq R} r^{-n} \int_{P_{r}\left(x, r^{2 m}\right)}\left|\nabla^{k} \hat{u}_{0}\right|^{\frac{2 m}{k}} \leq C\left\|u_{0}\right\|_{L^{\infty}\left(\mathbb{R}^{n}\right)}^{\frac{2 m}{k}-2} \cdot\left[u_{0}\right]_{\mathrm{BMO}_{R}\left(\mathbb{R}^{n}\right)}^{2},
$$

The proof of Lemma 3.1 is similar to 24 Lemma 3.1. For completeness, we sketch it here. Let $\mathcal{S}$ denote the class of Schwartz functions, the following characterization of BMO spaces, due to Carleson, is well-known (see, Stein [21]).

Lemma 3.2 For $0<R \leq+\infty$, let $\Phi \in \mathcal{S}$ be such that $\int_{\mathbb{R}^{n}} \Phi=0$ and denote for $t>0$, $\Phi_{t}(x)=t^{-n} \Phi\left(\frac{x}{t}\right), x \in \mathbb{R}^{n}$. If $f \in \mathrm{BMO}_{R}\left(\mathbb{R}^{n}\right)$, then

$$
\sup _{x \in \mathbb{R}^{n}, 0<r \leq R} r^{-n} \int_{0}^{r} \int_{B_{r}(x)}\left|\Phi_{t} * f\right|^{2}(x, t) \frac{d x d t}{t} \leq C\left[u_{0}\right]_{\mathrm{BMO}_{R}\left(\mathbb{R}^{n}\right)}
$$

for some $C=C(n)>0$.

Proof of Lemma 3.1. Let $g$ be given by (2.3) and $\Phi^{i}=\nabla^{i} g$ for $i=1, \cdots, m$. Then $\Phi^{i} \in \mathcal{S}$ and $\int_{\mathbb{R}^{n}} \Phi^{i}=0$ for $i=1, \cdots, m$. Direct calculations show

$$
\Phi_{t}^{i}(x)=t^{-n}\left(\nabla^{i} g\right)\left(\frac{x}{t}\right)=t^{i} \nabla^{i}\left(t^{-n} g\left(\frac{x}{t}\right)\right)=t^{i} \nabla^{i} g_{t}(x),
$$

where $g_{t}(x)=t^{-n} g\left(\frac{x}{t}\right)$. Hence we have

$$
\Phi_{t}^{i} * u_{0}(x)=t^{i} \nabla^{i}\left(g_{t} * u_{0}\right)(x) .
$$

Since the polyharmonic heat kernel $b(x, t)=g_{t \frac{1}{2 m}}(x)$, we have

$$
\Phi_{t}^{i} * u_{0}(x)=t^{i} \nabla^{i}\left[\left(b\left(\cdot, t^{2 m}\right) * u_{0}\right)(x)\right]=t^{i} \nabla^{i}\left(\mathbf{G} u_{0}\right)\left(x, t^{2 m}\right) .
$$

Thus Lemma 3.1 implies that for $i=1, \cdots, m$,

$$
\begin{aligned}
C\left[u_{0}\right]_{\mathrm{BMO}_{R}\left(\mathbb{R}^{n}\right)}^{2} & \geq \sup _{x \in \mathbb{R}^{n}, 0<r \leq R} r^{-n} \int_{0}^{r} \int_{B_{r}(x)}\left|\Phi_{t}^{i} * u_{0}\right|^{2} \frac{d x d t}{t} \\
& =\sup _{x \in \mathbb{R}^{n}, 0<r \leq R} r^{-n} \int_{0}^{r} \int_{B_{r}(x)} t^{2 i-1}\left|\nabla^{i} \mathbf{G} u_{0}\right|^{2}\left(x, t^{2 m}\right) d x d t \\
& =\frac{1}{2 m} \sup _{x \in \mathbb{R}^{n}, 0<r \leq R} r^{-n} \int_{P_{r}\left(x, r^{2 m}\right)} t^{\frac{2 i-2 m}{2 m}}\left|\nabla^{i} \mathbf{G} u_{0}\right|^{2}(x, t) d x d t .
\end{aligned}
$$


This clearly implies (3.1), since for $i=1, \cdots, m, t^{\frac{2 i-2 m}{2 m}} \geq r^{2 i-2 m}$ when $0<t \leq r^{2 m}$.

Since $\hat{u}_{0}$ solves the polyharmonic heat equation:

$$
\left(\partial_{t}+(-1)^{m} \Delta^{m}\right) \hat{u}_{0}=0 \text { on } \mathbb{R}^{n} \times(0,+\infty),
$$

the standard theory implies that for any $x \in \mathbb{R}^{n}$ and $r>0$,

$$
\sum_{k=1}^{m} r^{\frac{k}{m}}\left|\nabla^{k} \hat{u}_{0}\right|^{2}\left(x, r^{2 m}\right) \leq C \sum_{k=1}^{m} r^{-n} \int_{P_{r}\left(x, r^{2 m}\right)} r^{2 k-2 m}\left|\nabla^{k} \hat{u}_{0}\right|^{2} .
$$

Taking supremum over $x \in \mathbb{R}^{n}$ and $0<t=r^{2 m} \leq R^{2 m}$ yield (3.2).

For (3.3), observe that $u_{0} \in L^{\infty}\left(\mathbb{R}^{n}\right)$ implies $\Phi_{t}^{i} * u_{0} \in L^{\infty}\left(\mathbb{R}^{n}\right)$ for $i=1, \cdots, m-1$, and

$$
\left\|\Phi_{t}^{i} * u_{0}\right\|_{L^{\infty}\left(\mathbb{R}^{n}\right)} \leq\left\|\Phi^{i}\right\|_{L^{1}\left(\mathbb{R}^{n}\right)}\left\|u_{0}\right\|_{L^{\infty}\left(\mathbb{R}^{n}\right)} \leq\left\|\nabla^{i} g\right\|_{L^{1}\left(\mathbb{R}^{n}\right)}\left\|u_{0}\right\|_{L^{\infty}\left(\mathbb{R}^{n}\right)} \leq C\left\|u_{0}\right\|_{L^{\infty}\left(\mathbb{R}^{n}\right)} .
$$

Hence

$$
\begin{aligned}
& \sup _{x \in \mathbb{R}^{n}, 0<r \leq R} r^{-n} \int_{P_{r}\left(x, r^{2 m}\right)}\left|\nabla^{i} \mathbf{G} u_{0}\right|^{\frac{2 m}{i}} d x d t \\
& =\sup _{x \in \mathbb{R}^{n}, 0<r \leq R} r^{-n} \int_{B_{r}(x) \times[0, r]}\left|\Phi_{t}^{i} * u_{0}\right|^{\frac{2 m}{i}} \frac{d x d t}{t} \\
& \leq\left(\sup _{t>0}\left\|\Phi_{t}^{i} * u_{0}\right\|_{L^{\infty}\left(\mathbb{R}^{n}\right)}\right)_{L^{\infty}\left(\mathbb{R}^{n}\right)}^{\frac{2 m}{i}-2} \cdot \sup _{x \in \mathbb{R}^{n}, 0<r \leq R} r^{-n} \int_{B_{r}(x) \times[0, r]}\left|\Phi_{t}^{i} * u_{0}\right|^{2} \frac{d x d t}{t} \\
& \leq C\left\|u_{0}\right\|_{L^{\infty}\left(\mathbb{R}^{n}\right)}^{\frac{2 m}{i}-2} \cdot\left[u_{0}\right]_{\mathrm{BMO}_{R}\left(\mathbb{R}^{n}\right)}^{2}
\end{aligned}
$$

This implies (3.3).

Now we prove an important estimate on the distance of $\hat{u}_{0}$ to the manifold $N$ in term of the BMO norms of $u_{0}$. More precisely,

Lemma 3.3 For any $\delta>0$, there exists $K_{0}=K_{0}(\delta, N)>0$ such that for $0<R \leq+\infty$, if $u_{0} \in \mathrm{BMO}_{R}\left(\mathbb{R}^{n}\right)$ then

$$
\operatorname{dist}\left(\hat{u}_{0}(x, t), N\right) \leq K_{0}\left[u_{0}\right]_{\mathrm{BMO}_{R}\left(\mathbb{R}^{n}\right)}+\delta, \forall x \in \mathbb{R}^{n}, 0 \leq t \leq\left(\frac{R}{K_{0}}\right)^{2 m} .
$$

Proof. For any $x \in \mathbb{R}^{n}, t>0$ and $K>0$, denote

$$
c_{x, t}^{K}=\frac{1}{\left|B_{K}(0)\right|} \int_{B_{K}(0)} u_{0}\left(x-t^{\frac{1}{2 m}} z\right) d z .
$$

Let $g$ be given by (2.3). Then, by a change of variables, we have

$$
\hat{u}_{0}(x, t)=\int_{\mathbb{R}^{n}} g(y) u_{0}\left(x-t^{\frac{1}{2 m}} y\right) d y .
$$


Applying (2.5) (with $L=n+1$ ) from Lemma 2.2, we have

$$
\begin{aligned}
&\left|\hat{u}_{0}(x, t)-c_{x, t}^{K}\right| \leq \int_{\mathbb{R}^{n}} g(y)\left|u_{0}\left(x-t^{\frac{1}{2 m}} y\right)-c_{x, t}^{K}\right| d y \\
& \leq\left\{\int_{B_{K}(0)}+\int_{\mathbb{R}^{n} \backslash B_{K}(0)}\right\} g(y)\left|u_{0}\left(x-t^{\frac{1}{2 m}} y\right)-c_{x, t}^{K}\right| d y \\
& \leq \int_{B_{K}(0)}\left|u_{0}\left(x-t^{\frac{1}{2 m}} y\right)-c_{x, t}^{K}\right| d y \\
&+C\left\|u_{0}\right\|_{L^{\infty}\left(\mathbb{R}^{n}\right)} \int_{\mathbb{R}^{n} \backslash B_{K}(0)} \frac{1}{|y|^{n+1}} d y \\
& \leq K^{n}\left[u_{0}\right]_{\mathrm{BMO}} \frac{1}{K t} \frac{1}{2 m}\left(\mathbb{R}^{n}\right) \\
&
\end{aligned}
$$

provided we choose a sufficiently large $K=K_{0}(\delta, N)>0$ so that

$$
C\left\|u_{0}\right\|_{L^{\infty}\left(\mathbb{R}^{n}\right)} \int_{\mathbb{R}^{n} \backslash B_{K}(0)} \frac{1}{|y|^{n+1}} d y \leq \delta .
$$

On the other hand, since $u_{0}\left(\mathbb{R}^{n}\right) \subset N$, we have

$$
\operatorname{dist}\left(c_{x, t}^{K}, N\right) \leq \frac{1}{\left|B_{K}(0)\right|} \int_{B_{K}(0)}\left|c_{x, t}^{K}-u_{0}\left(x-t^{\frac{1}{2 m}} y\right)\right| d y \leq\left[u_{0}\right]_{\mathrm{BMO}_{K t} \frac{1}{2 m}}\left(\mathbb{R}^{n}\right) .
$$

Putting (3.6) and (3.7) together yields (3.5) holds for $t \leq\left(\frac{R}{K}\right)^{2 m}$.

\section{Boundedness of the operator $\mathrm{S}$}

In this section, we introduce several function spaces and establish the boundedness of the operator $\mathbf{S}$ between these spaces.

For $0<T<\infty$, the spaces $Y_{T}^{k}$, for $k=0, \cdots, m-1$, are the sets consisting of all functions $f: \mathbb{R}^{n} \times[0, T] \rightarrow \mathbb{R}$ such that

$$
\|f\|_{Y_{T}^{k}}:=\sup _{0<t \leq T} t^{\frac{2 m-k}{2 m}}\|f\|_{L^{\infty}\left(\mathbb{R}^{n}\right)}+\sup _{x \in \mathbb{R}^{n}, 0<R \leq T}\left(R^{-n} \int_{P_{R}(x)}|f|^{\frac{2 m}{2 m-k}}\right)^{\frac{2 m-k}{2 m}} .
$$

Notice that $\left(Y_{T}^{k},\|\cdot\|_{Y_{T}^{k}}\right)$ is a Banach space for $k=0, \cdots, m-1$. When $T=+\infty$, we simply denote $\left(Y^{k},\|\cdot\|_{Y^{k}}\right)$ for $\left(Y_{\infty}^{k},\|\cdot\|_{Y_{\infty}^{k}}\right)$.

Let the operator $\mathbf{S}$ be defined by (2.12). Then we have

Lemma 4.1 For any $0<T \leq+\infty$ and $k=0, \cdots, m-1$, if $f \in Y_{T}^{k}$, then $\mathbf{S}\left(\nabla^{\alpha} f\right) \in X_{T}$ and

$$
\left\|\mathbf{S}\left(\nabla^{\alpha} f\right)\right\|_{X_{T}} \leq C\|f\|_{Y_{T}^{k}}
$$

where $\alpha=\left(\alpha_{1}, \cdots \alpha_{n}\right)$ is any multi-index of order $k$. 
Proof. We need to show the point wise estimate

$$
\sum_{i=0}^{m} R^{i}\left|\nabla^{i} \mathbf{S}\left(\nabla^{\alpha} f\right)\right|\left(x, R^{2 m}\right) \leq C\|f\|_{Y_{T}^{k}}, \quad \forall x \in \mathbb{R}^{n}, \quad 0<R \leq T^{\frac{1}{2 m}},
$$

and the integral estimate for $0<R \leq T^{\frac{1}{2 m}}$ :

$$
\sum_{i=1}^{m} R^{-\frac{i n}{2 m}}\left\|\nabla^{i} \mathbf{S}\left(\nabla^{\alpha} f\right)\right\|_{L^{\frac{2 m}{i}}\left(P_{R}\left(x, R^{2 m}\right)\right)} \leq C\|f\|_{Y_{T}^{k}}
$$

By suitable scaling, we may assume $T \geq 1$. Since both estimates are translation and scale invariant, it suffices to show (4.3) and (4.4) hold for $x=0$ and $R=1$.

For $i=0, \cdots, m$ and $\alpha=\left(\alpha_{1}, \cdots, \alpha_{n}\right)$ with order $k$, we have

$$
\begin{aligned}
\left|\nabla^{i} \mathbf{S}\left(\nabla^{\alpha} f\right)\right|(0,1) & =\left|\int_{0}^{1} \int_{\mathbb{R}^{n}} \nabla^{i+\alpha} b(y, 1-s) f(y, s) d y d s\right| \\
& \leq\left\{\int_{\frac{1}{2}}^{1} \int_{\mathbb{R}^{n}}+\int_{0}^{\frac{1}{2}} \int_{B_{2}}+\int_{0}^{\frac{1}{2}} \int_{\mathbb{R}^{n} \backslash B_{2}}\right\}\left|\nabla^{i+k} b(y, 1-s)\right||f(y, s)| d y d s \\
& =I_{1}+I_{2}+I_{3} .
\end{aligned}
$$

Applying Lemma 2.2, we can estimate $I_{1}, I_{2}$ and $I_{3}$ as follows.

$$
\begin{aligned}
\left|I_{1}\right| & \leq\left(\sup _{\frac{1}{2} \leq s \leq 1}\|f(s)\|_{L^{\infty}\left(\mathbb{R}^{n}\right)}\right)\left(\int_{\frac{1}{2}}^{1}\left\|\nabla^{i+k} b(\cdot, 1-s)\right\|_{L^{1}\left(\mathbb{R}^{n}\right)} d s\right) \\
& \leq C\|f\|_{Y_{1}^{k}} \int_{0}^{\frac{1}{2}} s^{-\frac{i+k}{2 m}} d s \quad(\text { by }(2.7)) \\
& \leq C\|f\|_{Y_{1}^{k}}(\text { since } i+k \leq 2 m-1) . \\
\left|I_{2}\right| \leq & \left(\sup _{0 \leq s \leq \frac{1}{2}}\left\|\nabla^{i+k} b(\cdot, 1-s)\right\|_{L^{\infty}\left(\mathbb{R}^{n}\right)}\right)\left(\int_{B_{2} \times\left[0, \frac{1}{2}\right]}|f(y, s)| d y d s\right) \\
\leq & C \int_{B_{2} \times\left[0, \frac{1}{2}\right]}|f(y, s)| d y d s \\
\leq & C\|f\|_{Y_{1}^{k} .} \\
\left|I_{3}\right| \leq & \int_{0}^{\frac{1}{2}} \int_{\mathbb{R}^{n} \backslash B_{2}}\left|\nabla^{i+k} b(y, 1-s)\right||f(y, s)| d y d s \\
\leq & C \int_{0}^{\frac{1}{2}} \int_{\mathbb{R}^{n} \backslash B_{2}}|y|^{-(n+1)}|f(y, s)| d y d s \quad(\text { by }(2.6) \text { for } L=n+1) \\
\leq & \left(\sum_{k=2}^{\infty} k^{n-1} k^{-(n+1)}\right)\left(\sup _{x \in \mathbb{R}^{n}} \int_{P_{1}(x, 1)}|f(y, s)| d y d s\right) \\
\leq & C\left(\sum_{k=2}^{\infty} k^{-2}\right)\|f\|_{Y_{1}^{k}} \leq\|f\|_{Y_{1}^{k} .}
\end{aligned}
$$


Now we want to show (4.4) by the energy method. Denote $w=\mathbf{S}\left(\nabla^{\alpha} f\right)$. Then $w$ solves

$$
\left(\partial_{t}+(-1)^{m} \Delta^{m}\right) w=\nabla^{\alpha} f \quad \text { in } \mathbb{R}^{n} \times(0,+\infty) ;\left.\quad w\right|_{t=0}=0 .
$$

Let $\eta \in C_{0}^{\infty}\left(B_{2}\right)$ be a cut-off function of $B_{1}$. Multiplying (4.5) by $\eta^{4} w$ and integrating over $\mathbb{R}^{n} \times[0,1]$, we obtain

$$
\int_{\mathbb{R} \times\{1\}}|w|^{2} \eta^{4}+2 \int_{\mathbb{R}^{n} \times[0,1]} \nabla^{m} w \cdot \nabla^{m}\left(w \eta^{4}\right)=2 \int_{\mathbb{R}^{n} \times[0,1]} \nabla^{\alpha} f \cdot w \eta^{4} .
$$

By the Hölder inequality, we have

$$
\begin{aligned}
& \int_{\mathbb{R}^{n} \times[0,1]} \nabla^{m} w \cdot \nabla^{m}\left(w \eta^{4}\right) \\
= & \int_{\mathbb{R}^{n} \times[0,1]}\left|\nabla^{m}\left(w \eta^{2}\right)\right|^{2}+\int_{\mathbb{R}^{n} \times[0,1]} \nabla^{m} w\left(\sum_{\beta=0}^{m-1} \nabla^{\beta}\left(w \eta^{2}\right) \cdot \nabla^{m-\beta}\left(\eta^{2}\right)\right) \\
& -\int_{\mathbb{R}^{n} \times[0,1]} \nabla^{m}\left(w \eta^{2}\right)\left(\sum_{\beta=0}^{m-1} \nabla^{\beta}(w) \cdot \nabla^{m-\beta}\left(\eta^{2}\right)\right) \\
\geq & \frac{1}{2} \int_{\mathbb{R}^{n} \times[0,1]}\left|\nabla^{m}\left(w \eta^{2}\right)\right|^{2}-C \sum_{\beta=0}^{m-1} \int_{B_{2} \times[0,1]}\left|\nabla^{\beta} w\right|^{2} \\
& \int_{\mathbb{R}^{n} \times[0,1]} \nabla^{\alpha} f \cdot w \eta^{4}=(-1)^{k} \int_{\mathbb{R}^{n} \times[0,1]} f \cdot \nabla^{\alpha}\left[\left(w \eta^{2}\right) \eta^{2}\right] \\
\leq & C \sum_{\beta=0}^{k} \int_{\mathbb{R}^{n} \times[0,1]}\left|f \| \nabla^{\beta}\left(w \eta^{2}\right)\right| \\
\leq & C \sum_{\beta=0}^{k-1} \sup _{0<t \leq 1} t^{\frac{2 m-k}{2 m}}\|f\|_{L^{\infty}\left(\mathbb{R}^{n}\right)} \cdot \sup _{0<t \leq 1} t_{\frac{\beta}{2 m}\left\|\nabla^{\beta} w\right\|_{L^{\infty}\left(\mathbb{R}^{n}\right)} \cdot \int_{0}^{1} t^{-1+\frac{k-\beta}{2 m}} d t} \cdot\left\|\nabla^{k}\left(w \eta^{2}\right)\right\|_{L^{\frac{2 m}{k}}\left(\mathbb{R}^{n} \times[0,1]\right)} \\
& +C\|f\|_{L^{\frac{2 m}{2 m-k}}\left(B_{2} \times[0,1]\right)} \\
\leq & C\|f\|_{Y_{1}^{k}}^{2}+C\|f\|_{Y_{1}^{k}} \cdot\left\|\nabla^{k}\left(w \eta^{2}\right)\right\|_{L^{\frac{2 m}{k}}\left(\mathbb{R}^{n} \times[0,1]\right)}
\end{aligned}
$$

To estimate the last term, we need the Nirenberg interpolation inequality: for $k \leq m-1$,

$$
\left\|\nabla^{k}\left(w \eta^{2}\right)\right\|_{L^{\frac{2 m}{k}}\left(\mathbb{R}^{n}\right)}^{\frac{2 m}{k}} \leq C\left\|w \eta^{2}\right\|_{L^{\infty}\left(\mathbb{R}^{n}\right)}^{\frac{2 m}{k}-2}\left\|\nabla^{m}\left(w \eta^{2}\right)\right\|_{L^{2}\left(\mathbb{R}^{n}\right)}^{2}
$$

which, after integrating with respect to $t \in[0,1]$, implies

$$
\left\|\nabla^{k}\left(w \eta^{2}\right)\right\|_{L^{\frac{2 m}{k}}\left(\mathbb{R}^{n} \times[0,1]\right)} \leq C \sup _{0 \leq t \leq 1}\|w\|_{L^{\infty}\left(\mathbb{R}^{n}\right)}^{1-\frac{k}{m}}\left\|\nabla^{m}\left(w \eta^{2}\right)\right\|_{L^{2}\left(\mathbb{R}^{n} \times[0,1]\right)}^{\frac{k}{m}}
$$

Putting (4.9), (4.7) and (4.8) into (4.6), we have 


$$
\begin{aligned}
& \int_{\mathbb{R}^{n} \times[0,1]}\left|\nabla^{m}\left(w \eta^{2}\right)\right|^{2} \\
\leq & C \sum_{\beta=0}^{m-1} \int_{B_{2} \times[0,1]}\left|\nabla^{\beta} w\right|^{2}+C\|f\|_{Y_{1}^{k}}^{2}+C\|f\|_{Y_{1}^{k}} \cdot\left\|\nabla^{k}\left(w \eta^{2}\right)\right\|_{L^{\frac{2 m}{k}}\left(\mathbb{R}^{n} \times[0,1]\right)} \\
\leq & C \sum_{\beta=0}^{m-1}\left[\int_{0}^{1} t^{-\frac{\beta}{m}} d t \cdot \sup _{0<t \leq 1}\left(t^{\frac{\beta}{m}}\left\|\nabla^{\beta} w(t)\right\|_{L^{\infty}\left(\mathbb{R}^{n}\right)}^{2}\right)\right] \\
& +C\|f\|_{Y_{1}^{k}}^{2}+C\|f\|_{Y_{1}^{k}} \sup _{0 \leq t \leq 1}\|w\|_{L^{\infty}\left(\mathbb{R}^{n}\right)}^{1-\frac{k}{m}}\left\|\nabla^{m}\left(w \eta^{2}\right)\right\|_{L^{2}\left(\mathbb{R}^{n} \times[0,1]\right)}^{\frac{k}{m}} \\
\leq & C\|f\|_{Y_{1}^{k}}^{2}+\frac{1}{2} \int_{\mathbb{R}^{n} \times[0,1]}\left|\nabla^{m}\left(w \eta^{2}\right)\right|^{2}+C\|f\|_{Y_{1}^{k}}^{q} \cdot\|w\|_{L^{\infty}\left(\mathbb{R}^{n}\right)}^{\left(1-\frac{k}{m}\right) q} \\
\leq & \frac{1}{2} \int_{\mathbb{R}^{n} \times[0,1]}\left|\nabla^{m}\left(w \eta^{2}\right)\right|^{2}+C\|f\|_{Y_{1}^{k}}^{2},
\end{aligned}
$$

where $q=\frac{2 m}{2 m-k}$. Therefore, we obtain

$$
\int_{P_{1}(0,1)}\left|\nabla^{m} w\right|^{2} \leq \int_{\mathbb{R}^{n} \times[0,1]}\left|\nabla^{m}\left(w \eta^{2}\right)\right|^{2} \leq C\|f\|_{Y_{1}^{k}}^{2} .
$$

For $i=1, \cdots, m-1$, applying Nirenberg's interpolation inequality gives

$$
\begin{aligned}
& \int_{P_{1}(0,1)}\left|\nabla^{i} w\right|^{\frac{2 m}{i}} \leq \int_{\mathbb{R}^{n} \times[0,1]}\left|\nabla^{i}\left(w \eta^{2}\right)\right|^{\frac{2 m}{i}} \\
\leq & \sup _{0 \leq t \leq 1}\|w\|_{L^{\infty}\left(\mathbb{R}^{n}\right)}^{\frac{2 m}{i}-2}\left\|\nabla^{m}\left(w \eta^{2}\right)\right\|_{L^{2}\left(\mathbb{R}^{n} \times[0,1]\right)}^{2} \\
\leq & C\|f\|_{Y_{1}^{k}}^{\frac{2 m}{i}-2} \cdot \int_{\mathbb{R}^{n} \times[0,1]}\left|\nabla^{m}\left(w \eta^{2}\right)\right|^{2} \\
\leq & C\|f\|_{Y_{1}^{k}}^{\frac{2 m}{i}}
\end{aligned}
$$

(4.11) and (4.12) imply (4.4). This completes the proof.

\section{Proof of Theorem 1.2}

This section is devoted to the proof of Theorem 1.2. The idea is based on the fixed point theorem in a small ball inside $X_{R^{2 m}}$.

Since the image of a map $u \in X_{R^{2 m}}$ may not be contained in $N$, we first need to extend $\Pi$ to $\mathbb{R}^{l}$, denoted as $\widetilde{\Pi}$, such that $\widetilde{\Pi} \in C^{\infty}\left(\mathbb{R}^{l}\right)$ and $\widetilde{\Pi} \equiv \Pi$ in $N_{\delta_{N}}$.

Let

$$
\begin{aligned}
\widetilde{F}(u):= & (-1)^{m} \operatorname{div}^{m}\left(\sum_{k=0}^{m-2}\left(\begin{array}{c}
m-1 \\
k
\end{array}\right) \nabla^{m-k-1}(\widetilde{\Pi}(u)) \nabla^{k+1} u\right) \\
& -\sum_{k=0}^{m-1}(-1)^{k}\left(\begin{array}{c}
m \\
k
\end{array}\right) \operatorname{div}^{k}\left(\nabla^{m-k}(\widetilde{\Pi}(u)) \nabla^{m} u\right) .
\end{aligned}
$$


For $k=0, \cdots, m-2$, define

$$
F_{k}(u)=(-1)^{k+1}\left(\begin{array}{c}
m \\
k
\end{array}\right) \nabla^{m-k}(\widetilde{\Pi}(u)) \nabla^{m} u .
$$

For $k=m-1$, define

$$
\begin{aligned}
& F_{m-1}(u) \\
= & (-1)^{m}\left(\begin{array}{c}
m \\
m-1
\end{array}\right) \nabla(\widetilde{\Pi}(u)) \nabla^{m} u \\
+ & (-1)^{m} \sum_{k=0}^{m-2}\left(\begin{array}{c}
m-1 \\
k
\end{array}\right)\left(\operatorname{div}\left(\nabla^{m-k-1}(\widetilde{\Pi}(u))\right) \nabla^{k+1} u+\nabla^{m-k-1}(\widetilde{\Pi}(u)) \operatorname{div}\left(\nabla^{k+1} u\right)\right) .
\end{aligned}
$$

Then (1.2) can be written as

$$
\left(\partial_{t}+(-1)^{m} \Delta^{m}\right) u=\sum_{k=0}^{m-1} \operatorname{div}^{k}\left(F_{k}(u)\right) .
$$

The first observation is

Lemma 5.1 For $0<R \leq+\infty$, if $u \in X_{R^{2 m}}$, then $F_{k}(u) \in Y_{R^{2 m}}^{k}$ for $k=0, \cdots, m-1$. Moreover, there exists $C>0$ depends on $N$ and $\|u\|_{L^{\infty}\left(\mathbb{R}^{n} \times\left[0, R^{2 m}\right]\right)}$ such that

$$
\left\|F_{k}(u)\right\|_{Y_{R^{2 m}}^{k}} \leq C \sum_{l=1}^{m}[u]_{X_{R^{2 m}}^{l}}^{\frac{2 m-k}{l}}, 0 \leq k \leq m-1 .
$$

Proof. It follows from direct calculations and Hölder inequality that

$$
\left|F_{k}(u)\right| \leq C \sum_{l=1}^{m}\left|\nabla^{l} u\right|^{\frac{2 m-k}{l}}, 0 \leq k \leq m-1,
$$

where $C>0$ depends on $N$ and $\|u\|_{L^{\infty}\left(\mathbb{R}^{n} \times\left[0, R^{2 m}\right]\right)}$. Thus we have, by the definitions of $X_{R^{2 m}}$ and $Y_{R^{2 m}}^{k}$, that

$$
\left\|F_{k}(u)\right\|_{Y_{R^{2 m}}^{k}} \leq C \sum_{l=1}^{m}[u]_{X_{R^{2 m}}^{l}}^{\frac{2 m-k}{l}}, \forall 0 \leq k \leq m-1 .
$$

This completes the proof.

By the Duhamel formula (2.10), the solution $u$ to (5.2) is given by

$$
u(x, t)=\mathbf{G} u_{0}+\sum_{k=0}^{m-1} \mathbf{S}\left(\operatorname{div}^{k}\left(F_{k}(u)\right)\right) .
$$

From now on,denote

$$
\hat{u}_{0}=\mathbf{G} u_{0} \text {. }
$$

Define the mapping operator $\mathbf{T}$ on $X_{R^{2 m}}$ by letting

$$
\mathbf{T} u(x, t)=\hat{u}_{0}+\sum_{k=0}^{m-1} \mathbf{S}\left(\operatorname{div}^{k}\left(F_{k}(u)\right)\right) .
$$

The following property follows directly from Lemma 3.1 . 
Lemma 5.2 For any $0<R \leq+\infty$, if $u_{0}: \mathbb{R}^{n} \rightarrow N$, then $\hat{u}_{0} \in X_{R^{2 m}}$, and

$$
\left\|\hat{u}_{0}\right\|_{L^{\infty}\left(\mathbb{R}^{n} \times\left[0, R^{2 m}\right]\right)} \leq C\left\|u_{0}\right\|_{L^{\infty}\left(\mathbb{R}^{n}\right)}, \quad\left[\hat{u}_{0}\right]_{X_{R^{2 m}}} \leq C\left[u_{0}\right]_{\mathrm{BMO}_{R}\left(\mathbb{R}^{n}\right)}
$$

For any $\varepsilon>0$ and $0<R \leq+\infty$, let

$$
\mathbf{B}_{\varepsilon}\left(\hat{u}_{0}\right):=\left\{u \in X:\left\|u-\hat{u}_{0}\right\|_{X_{R^{2 m}}} \leq \varepsilon\right\}
$$

be the ball in $X_{R^{2 m}}$ with center $\hat{u}_{0}$ and radius $\varepsilon$. By the triangle inequality, we have

$$
\|u\|_{L^{\infty}\left(\mathbb{R}^{n} \times\left[0, R^{2 m}\right]\right)} \leq C\left\|u_{0}\right\|_{L^{\infty}\left(\mathbb{R}^{n}\right)}+\varepsilon,[u]_{X_{R^{2 m}}} \leq C\left[u_{0}\right]_{\mathrm{BMO}_{R}\left(\mathbb{R}^{n}\right)}+\varepsilon, \forall u \in \mathbf{B}_{\varepsilon}\left(\hat{u}_{0}\right) .
$$

Thus we have

Lemma 5.3 For $0<R \leq+\infty$, if $u_{0}: \mathbb{R}^{n} \rightarrow N$ has $\left[u_{0}\right]_{\mathrm{BMO}_{R}\left(\mathbb{R}^{n}\right)} \leq \varepsilon$, then

$$
\|u\|_{L^{\infty}\left(\mathbb{R}_{+}^{n+1}\right)} \leq C+\varepsilon, \quad[u]_{X} \leq C \varepsilon, \quad \forall u \in \mathbf{B}_{\varepsilon}\left(\hat{u}_{0}\right),
$$

for some $C=C(n, N)>0$.

The proof of Theorem 1.2 is based on the following two lemmas.

Lemma 5.4 There exists $\varepsilon_{1}>0$ such that for $0<R \leq+\infty$, if $u_{0}: \mathbb{R}^{n} \rightarrow N$ has

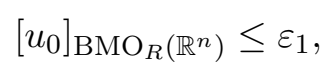

then $\mathbf{T}$ maps $\mathbf{B}_{\varepsilon_{1}}\left(\hat{u}_{0}\right)$ to $\mathbf{B}_{\varepsilon_{1}}\left(\hat{u}_{0}\right)$.

Proof. By (5.5), we have

$$
\mathbf{T}(u)-\hat{u}_{0}=\sum_{k=0}^{m-1} \mathbf{S}\left(\operatorname{div}^{k}\left(F_{k}(u)\right)\right), \quad u \in \mathbf{B}_{\varepsilon_{1}}\left(\hat{u}_{0}\right) .
$$

Hence Lemma 4.1, Lemma 5.1 and Lemma 5.2 imply that for any $u \in \mathbf{B}_{\varepsilon_{1}}\left(\hat{u}_{0}\right)$,

$$
\begin{aligned}
\left\|\mathbf{T}(u)-\hat{u}_{0}\right\|_{X_{R^{2 m}}} & \lesssim \sum_{k=0}^{m-1}\left\|\mathbf{S}\left(\operatorname{div}^{k}\left(F_{k}(u)\right)\right)\right\|_{X_{R^{2 m}}} \\
& \lesssim \sum_{k=0}^{m-1}\left\|F_{k}(u)\right\|_{Y_{R^{2 m}}^{k}} \\
& \lesssim \sum_{k=0}^{m-1} \sum_{l=1}^{m}[u]_{X_{R^{2 m}}^{\frac{2 m-k}{l}}} \\
& \leq C[u]_{X_{R^{2 m}}^{\frac{m+1}{m}}} \leq \varepsilon_{1},
\end{aligned}
$$

provide $\varepsilon_{1}>0$ is chosen to be sufficiently small. Hence $\mathbf{T} u \in \mathbf{B}_{\varepsilon_{1}}\left(\hat{u}_{0}\right)$. This completes the proof. 
Lemma 5.5 There exist $0<\varepsilon_{2} \leq \varepsilon_{1}$ and $\theta_{0} \in(0,1)$ such that for $0<R \leq+\infty$, if $u_{0}: \mathbb{R}^{n} \rightarrow N$ satisfies

$$
\left[u_{0}\right]_{\mathrm{BMO}_{R}\left(\mathbb{R}^{n}\right)} \leq \varepsilon_{2},
$$

then $\mathbf{T}: \mathbf{B}_{\varepsilon_{2}}\left(\hat{u}_{0}\right): \rightarrow \mathbf{B}_{\varepsilon_{2}}\left(\hat{u}_{0}\right)$ is a $\theta_{0}$-contraction map, i.e.

$$
\|\mathbf{T}(u)-\mathbf{T}(v)\|_{X_{R^{2 m}}} \leq \theta_{0}\|u-v\|_{X_{R^{2 m}}}, \quad \forall u, v \in \mathbf{B}_{\varepsilon_{2}}\left(\hat{u}_{0}\right)
$$

Proof. For $u, v \in \mathbf{B}_{\varepsilon_{2}}\left(\hat{u}_{0}\right)$, we have

$$
\begin{aligned}
\|\mathbf{T}(u)-\mathbf{T}(v)\|_{X_{R^{2 m}}} & \leq \sum_{k=0}^{m-1}\left\|\mathbf{S}\left(\operatorname{div}^{k}\left(F_{k}(u)-F_{k}(v)\right)\right)\right\|_{X_{R^{2 m}}} \\
& \lesssim \sum_{k=0}^{m-1}\left\|F_{k}(u)-F_{k}(v)\right\|_{Y_{R^{2 m}}^{k}}
\end{aligned}
$$

Notice that $\|u\|_{L^{\infty}\left(\mathbb{R}^{n} \times\left[0, R^{2 m}\right]\right)}+\|v\|_{L^{\infty}\left(\mathbb{R}^{n} \times\left[0, R^{2 m}\right]\right)} \leq C_{0}$. For any $k=0, \cdots, m-2$, it follows from the definition of $F_{k}(u)$ we have

$$
\begin{aligned}
& \left|F_{k}(u)-F_{k}(v)\right| \\
\lesssim & \left|\nabla^{m}(u-v)\right|\left[\left|\nabla^{m-k} u\right|+\sum_{j=1}^{m-k}\left(\sum_{|\alpha|=j}\left(\Pi_{i=1}^{n}\left|\nabla^{\alpha_{i}} u\right|\right)\right)\left|\nabla^{m-k-j} u\right|\right] \\
+ & \left|\nabla^{m} v\right||u-v|\left[\sum_{|\alpha|=m-k}\left(\Pi_{i=1}^{n}\left|\nabla^{\alpha_{i}} u\right|+\Pi_{i=1}^{n}\left|\nabla^{\alpha_{i}} v\right|\right)\right] \\
+ & \left|\nabla^{m} v\right|\left[\sum_{j=1}^{m-k}\left|\nabla^{j}(u-v)\right|\left(\sum_{|\alpha|=m-k-j}\left(\Pi_{i=1}^{n}\left|\nabla^{\alpha_{i}} u\right|+\Pi_{i=1}^{n}\left|\nabla^{\alpha_{i}} v\right|\right)\right)\right] .
\end{aligned}
$$

Hence we have

$$
\begin{aligned}
\left\|F_{k}(u)-F_{k}(v)\right\|_{Y_{R^{2 m}}^{k}} & \lesssim\left[\sum_{j=1}^{m-k}\left([u]_{X_{R^{2 m}}}^{j}+[v]_{X_{R^{2 m}}}^{j}\right)\right]\|u-v\|_{X_{R^{2 m}}} \\
& \leq C \varepsilon_{2}\|u-v\|_{X_{R^{2 m}}},
\end{aligned}
$$

where we have used Lemma 5.3 in the last step.

For $k=m-1$, since

$$
\begin{aligned}
\mid F_{m-1}(u)-F_{m-1}(v) & \lesssim\left[|\nabla u|\left|\nabla^{m}(u-v)\right|+|\nabla(u-v)|\left|\nabla^{m} v\right|\right] \\
& +\sum_{k=0}^{m-2}\left[\left|\nabla^{m-k}(\widetilde{\Pi}(u))\right|+\left|\nabla^{m-k}(\widetilde{\Pi}(v))\right|\right]\left|\nabla^{k+1}(u-v)\right| \\
& +\sum_{k=0}^{m-2}\left|\nabla^{m-k}(\widetilde{\Pi}(u)-\widetilde{\Pi}(v))\right|\left[\left|\nabla^{k+1} u\right|+\left|\nabla^{k+1} v\right|\right],
\end{aligned}
$$


we also have

$$
\left\|F_{m-1}(u)-F_{m-1}(v)\right\|_{Y_{R^{2 m}}^{m-1}} \leq C \varepsilon_{2}\|u-v\|_{X_{R^{2 m}}} .
$$

Putting (5.10) and (5.11) into (5.9), we obtain

$$
\|\mathbf{T}(u)-\mathbf{T}(v)\|_{X_{R^{2 m}}} \leq C \varepsilon_{2}\|u-v\|_{X_{R^{2 m}}} \leq \theta_{0}\|u-v\|_{X_{R^{2 m}}}
$$

for some $\theta_{0}=\theta_{0}\left(\varepsilon_{2}\right) \in(0,1)$, provided $\varepsilon_{2}>0$ is chosen to be sufficiently small. This completes the proof of Lemma 5.5 .

Proof of Theorem 1.2, It follows from Lemma 5.4 and Lemma 5.5, and the fixed point theorem that there exists $\varepsilon_{0}>$ such that for $0<R \leq+\infty$, if $\left[u_{0}\right]_{\mathrm{BMO}_{R}\left(\mathbb{R}^{n}\right)} \leq \varepsilon_{0}$, then there exists a unique $u \in X_{R^{2 m}}$ such that

$$
u=\hat{u}_{0}+\mathbf{S}(\widetilde{F}(u)) \quad \text { on } \mathbb{R}^{n} \times\left[0, R^{2 m}\right],
$$

or equivalently

$$
u_{t}+(-1)^{m} \Delta^{m} u=\widetilde{F}(u) \quad \text { on } \mathbb{R}^{n} \times\left(0, R^{2 m}\right] ;\left.u\right|_{t=0}=u_{0} .
$$

We want to show $u\left(\mathbb{R}^{n} \times\left[0, R^{2 m}\right]\right) \subset N$. By Lemma 3.3, we have that for any $x \in \mathbb{R}^{n}$ and $0 \leq t \leq\left(\frac{R}{K_{0}}\right)^{2 m}$,

$$
\begin{aligned}
\operatorname{dist}(u(x, t), N) & \leq \operatorname{dist}\left(\hat{u}_{0}, N\right)+\left\|u-\hat{u}_{0}\right\|_{L^{\infty}\left(\mathbb{R}^{n} \times\left[0, R^{2 m}\right]\right)} \\
& \leq \delta+K_{0}\left[u_{0}\right]_{\mathrm{BMO}_{R}\left(\mathbb{R}^{n}\right)}+\varepsilon_{0} \\
& \leq \delta+\left(1+K_{0}\right) \varepsilon_{0} \leq \delta_{N}
\end{aligned}
$$

provided $\delta \leq \frac{\delta_{N}}{2}$ and $\varepsilon_{0}=\frac{\delta_{N}}{2\left(1+K_{0}\right)}$. This yields $u\left(\mathbb{R}^{n} \times\left[0,\left(\frac{R}{K_{0}}\right)^{2 m}\right]\right) \subset N_{\delta_{N}}$. Hence

$$
\widetilde{\Pi}(u)=\Pi(u), \quad \widetilde{F}(u)=F(u) \text { on } \mathbb{R}^{n} \times\left[0,\left(\frac{R}{K_{0}}\right)^{2 m}\right] .
$$

Set $Q(u)=y-\Pi(y)$ for $y \in N_{\delta_{N}}$, and $\rho(u)=\frac{1}{2}|Q(u)|^{2}$. Then direct calculations imply that for any $y \in N_{\delta_{N}}$,

$$
\begin{aligned}
\nabla Q(y)(v) & =(\operatorname{Id}-\nabla \Pi(y))(v), \forall v \in \mathbb{R}^{l} \\
\nabla^{2} Q(y)(v, w) & =-\nabla^{2} \Pi(y)(v, w), \forall v, w \in \mathbb{R}^{l} .
\end{aligned}
$$

Set $A(y)(v, w)=-\nabla^{2} \Pi(y)(v, w)$ for $y \in N_{\delta_{N}}$ and $v, w \in \mathbb{R}^{l}$. Then $F(u)$ can be rewritten by (see Gastel [8]):

$$
F(u)=(-1)^{m+1} \sum_{j=0}^{2 m-2} \sum_{|\alpha|=2 m-2-j}\left(\begin{array}{c}
2 m-2-j \\
\alpha
\end{array}\right) \operatorname{tr}^{m}\left(\nabla^{j} A\right) \circ u\left(\nabla^{\alpha_{1}+1} u, \nabla^{\alpha_{2}+1} u, \cdots, \nabla^{\alpha_{j+2}+1} u\right) .
$$

Direct calculations imply 


$$
\begin{aligned}
& \Delta^{m} Q(u)=\nabla Q(u)\left(\Delta^{m} u\right) \\
+ & \sum_{j=0}^{2 m-2} \sum_{|\alpha|=2 m-2-j}\left(\begin{array}{c}
2 m-2-j \\
\alpha
\end{array}\right) \operatorname{trace}^{m}\left(\nabla^{j} A\right) \circ u\left(\nabla^{\alpha_{1}+1} u, \nabla^{\alpha_{2}+1} u, \cdots, \nabla^{\alpha_{j+2}+1} u\right) \\
= & \nabla Q(u)\left(\Delta^{m} u\right)+(-1)^{m+1} F(u) .
\end{aligned}
$$

Therefore we have

$$
\begin{aligned}
\left(\partial_{t}+(-1)^{m} \Delta^{m}\right) Q(u) & =[\nabla Q(u) F(u)-F(u)] \\
& =-\nabla \Pi(u)(F(u)) .
\end{aligned}
$$

Multiplying both sides of (5.12) by $Q(u)$ and integrating over $\mathbb{R}^{n}$, we obtain that for $0 \leq t \leq\left(\frac{R}{K_{0}}\right)^{2 m}$,

$$
\left.\frac{d}{d t} \int_{\mathbb{R}^{n}} \rho(u)+\int_{\mathbb{R}^{n}}\left|\nabla^{m} Q(u)\right|^{2}=-\int_{\mathbb{R}^{n}}\left\langle\nabla \Pi_{(} u\right)(F(u)), Q(u)\right\rangle=0,
$$

where we have used the fact that $Q(u) \perp T_{\Pi(u)} N$ and $\nabla \Pi(u)(F(u)) \in T_{\Pi(u)} N$ on $\mathbb{R}^{n} \times$ $\left[0,\left(\frac{R}{K_{0}}\right)^{2 m}\right]$ in the last step.

Since $\left.\rho(u)\right|_{t=0}=0$, integrating (5.13) with respect to $t$ implies $\rho(u) \equiv 0$ on $\mathbb{R}^{n} \times$ $\left[0,\left(\frac{R}{K_{0}}\right)^{2 m}\right]$. Thus $u\left(\mathbb{R}^{n} \times\left[0,\left(\frac{R}{K_{0}}\right)^{2 m}\right]\right) \subset N$. Repeating the same argument also implies that $u\left(\mathbb{R}^{n} \times\left[\left(\frac{R}{K_{0}}\right)^{2 m}, R^{2 m}\right]\right) \subset N$. This completes the proof of Theorem 1.2 .

\section{References}

[1] G. Angelsberg, D. Pumberger, A regularity result for polyharmonic maps with higher integrability. Ann. Global Anal. Geom. 35 (2009), no. 1, 63-81.

[2] Y. Chen, W. Ding, Blow-up and global existence for heat flows of harmonic maps. Invent. Math. 99 (1990), no. 3, 567-578.

[3] K. Chang, W. Ding, R. Ye, Finite-time blow-up of the heat flow of harmonic maps from surfaces. J. Differen. Geom. 36(2), 507-515 (1992).

[4] J. Coron, J. Ghidaglia, Explosion en temps fini pour le flot des applications harmoniques. C.R. Acad. Sci. Paris 308, Serie I 339-344 (1989).

[5] Y. Chen, F. Lin, Evolution of harmonic maps with Dirichlet boundary conditions. Comm. Anal. Geom. 1 (1993), no. 3-4, 327-346..

[6] Y. Chen, M. Struwe, Existence and partial regularity for heat flow for harmonic maps. Math. Z. 201, 83-103 (1989).

[7] J. Eells, J. Sampson, Harmonic mappings of Riemannian manifolds. Amer. J. Math. 86, 109-160 (1964)

[8] A. Gastel, The extrinsic polyharmonic map heat flow in the critical dimension. Adv. Geom. 6 (2006), no. 4, 501-521. 
[9] A. Gastel, C. Scheven, Regularity of polyharmonic maps in the critical dimension. Comm. Anal. Geom. 17 (2009), no. 2, 185-226.

[10] P. Goldstein, P. Strzelecki, A. Zatorska-Goldstein, On polyharmonic maps into spheres in the critical dimension. Ann. Inst. H. Poincaré Anal. Non Linéaire, vol. 26, issue 4, pp. 1387-1405.

[11] S. Hildebrandt, H. Kaul, K. Widman, An existence theorem for harmonic mappings of Riemannian manifolds. Acta Math. 138 (1977), no. 1-2, 1-16.

[12] L. Hörmander, The analysis of linear partial differential operators I, 2nd edition, Springer-Verlag Berlin Heidelberg, 1990.

[13] H. Koch, T. Lamm, Geometric flows with rough initial data. arXiv: 0902.1488v1, 2009 .

[14] H. Koch, D. Tataru, Well-posedness for the Navier-Stokes equations. Adv. Math. 157 (2001), no. 1, 22-35.

[15] T. Lamm, Biharmonischer Wärmefluss. Diplomarbeit Universität Freiburg (2001).

[16] T. Lamm, Heat flow for extrinsic biharmonic maps with small initial energy. Ann. Global Anal. Geom. 26 (2004), no. 4, 369-384.

[17] T. Lamm, Biharmonic map heat flow into manifolds of nonpositive curvature. Calc. Var. Partial Differential Equations 22 (2005), no. 4, 421-445.

[18] T. Lamm, C. Wang, Boundary regularity for polyharmonic maps in the critical dimension. Adv. Calc. Var. 2 (2009), no. 1, 1-16.

[19] F. Lin, C. Wang, The analysis of harmonic maps and their heat flows. World Scientific Publishing Co. Pte. Ltd., Hackensack, NJ, 2008.

[20] R. Moser, Regularity of minimizing extrinsic polyharmonic maps in the critical dimension. Preprint (2009).

[21] E. Stein, Harmonic analysis, Vol. 43 of Princeton Mathematical Series, Princeton University Press, Princeton, NJ, 1993.

[22] M. Struwe, On the evolution of harmonic maps of Riemannian surfaces. Comment. Math. Helv. 60, 558-581 (1985).

[23] C. Wang, Heat flow of biharmonic maps in dimensions four and its application. Pure Appl. Math. Q. 3 (2007), no. 2, part 1, 595-613.

[24] C. Wang, Well-posedness for the heat flow of biharmonic maps with rough initial data. Preprint (2010).

[25] C. Wang, Well-posedness for the heat flow of harmonic maps and the liquid crystal flow with rough initial data. Preprint (2010). 